\title{
Duplicated zebrafish co-orthologs of parathyroid hormone-related peptide (PTHrP, Pthlh) play different roles in craniofacial skeletogenesis
}

\author{
Yi-Lin Yan, Poulomi Bhattacharya ${ }^{1}$, Xin Jun He, Bhaskar Ponugoti ${ }^{1}$, Ben Marquardt ${ }^{1}$, Jason Layman ${ }^{1}$, \\ Melissa Grunloh ${ }^{1}$, John H Postlethwait and David A Rubin ${ }^{1}$
}

Institute of Neuroscience, University of Oregon, Eugene, Oregon 97403, USA

${ }^{1}$ Department of Biological Sciences, Illinois State University, Normal, Illinois 61790, USA

(Correspondence should be addressed to D A Rubin; Email: adavid@ilstu.edu)

\begin{abstract}
In mammals, parathyroid hormone-related peptide (PTHrP, alias PTH-like hormone (Pthlh)) acts as a paracrine hormone that regulates the patterning of cartilage, bone, teeth, pancreas, and thymus. Beyond mammals, however, little is known about the molecular genetic mechanisms by which Pthlh regulates early development. To evaluate conserved pathways of craniofacial skeletogenesis, we isolated two Pthlh co-orthologs from the zebrafish (Danio rerio) and investigated their structural, phylogenetic, and syntenic relationships, expression, and function. Results showed that pthlh duplicates originated in the teleost genome duplication. Zebrafish pththa and pthlhb were maternally expressed and showed overlapping and distinct zygotic expression patterns during skeletal development that mirrored mammalian expression domains. To explore the regulation of duplicated pthlh genes, we
\end{abstract}

studied their expression patterns in mutants and found that both $\operatorname{sox} 9 a$ and sox $9 b$ are upstream of pthlha in arch and fin bud cartilages, but only sox $9 b$ is upstream of pthlha in the pancreas. Morpholino antisense knockdown showed that pthlha regulates both sox $9 a$ and sox $9 b$ in the pharyngeal arches but not in the brain or otic vesicles and that pthlhb does not regulate either sox 9 gene, which is likely related to its highly degraded nuclear localization signal. Knockdown of pthlha but not pthlhb caused runx $2 b$ overexpression in craniofacial cartilages and premature bone mineralization. We conclude that in normal cartilage development, sox 9 upregulates pthlh, which downregulates runx2, and that the duplicated nature of all three of these genes in zebrafish creates a network of regulation by different co-orthologs in different tissues.

Journal of Endocrinology (2012) 214, 421-435

\section{Introduction}

Parathyroid hormone (PTH) acts as the main hypercalcemic hormone while PTH-related protein (PTHrP, official human symbol PTHLH (PTH-like hormone) and referred to as Pthlh in this manuscript), is essential for embryonic development, differentiation, and tissue patterning (Philbrick et al. 1996). Unregulated paracrine secretion of Pthlh is associated with a type of tumor that results in elevated blood calcium levels, a condition called humoral hypercalcemia of malignancy (HHM), while regulated secretion of Pthlh during mouse embryogenesis is essential for the developmental patterning of cartilage, bone, teeth, CNS, pancreas, and other tissues (Karperien et al. 1996, Philbrick et al. 1996, Clemens et al. 2001).

Pthlh structure is highly conserved. For example, human, chicken, and rodent Pthlh are 98\% identical within the first 111 residues, while the average protein is only $85 \%$ identical compared with chickens and humans (Lagerström et al. 2006). Alternative splicing of human PTHLH transcripts produces three PTHLH variants that differ in their carboxy termini (Clemens et al. 2001, Sellers et al. 2004). Splice variants share a 'prepro' segment including the signal sequence, followed by 139 shared amino acids (Philbrick et al. 1996). The sequence of Pthlh, including the amino-terminus ( $\mathrm{N}$-terminus), mid-region, and nuclear localization signal (NLS) with its associated RNA-binding region, is remarkably conserved among species, suggesting functional conservation (Henderson et al. 1995, Wu et al. 1996; Fig. 1). Posttranslational processing produces forms with distinct physiological functions that work through unique yet poorly characterized receptors (Orloff et al. 1996, Valin et al. 2001) to regulate cartilage differentiation and apoptosis, chondrocyte metabolism, and matrix synthesis and to inhibit bone resorption (Clemens et al. 2001).

Mice with a disrupted Pthlh gene have a lethal chondrodysplasia characterized by abnormal growth plates with fewer resting and proliferative chondrocytes, premature endochondral and perichondral ossification, and death shortly after birth due to breathing complications. Mice chondrocytes overexpressing Pthlh display delayed chondrocyte differentiation in contrast to the premature chondrocyte differentiation syndrome caused by Pthlh knockdown (Karaplis et al. 1994). As a result, surviving mutant pups are born with a cartilaginous endochondral skeleton. After birth, 
A

PTHLH

MGRRLVOOWSVAVFLLSYAVPSCGRSVEGLSRRLKRAVSEHOLLHDKGKS IODLRRRFFLHHI Pthlha mrmLCCRRVLQQWVFALFLLCSPVPHHGRPVDALSSRMKR $\underline{S} V T H A Q L M H D K G R T L Q D F K R R M W L Q E L$

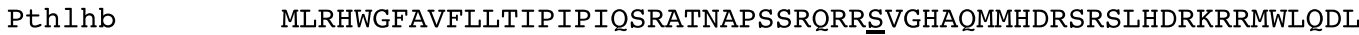

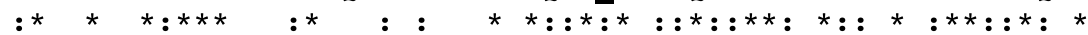
$+34$

PTHLH

Pthlha

Pthlhb

IAEIHTAEI

HPV

RFG

LHEVHTAEIreaqqprggvsissgagggvgaGVSitlpagvgvstgagtaHPKPAGGTKNLPIGFG LEQVHTAOVWDSPNQSEGSVQTLSwprpkhtGSTkniplgyqmesigtrdd : $: * * *$

Nuclear Localization Sequence

Mid-region RNA binding

PTHLH SDDE GRYLTQETNKVETYKEQPLKTPGKKKKGKP GKRKEQEKKKRRTRSawld SGVtgsgle Pthlha LEDEEGTNLPQETHKSQNYKDNMMKGIRRKKKSRT GKRREGEKRKRRARSLqefsSGVhlqpys

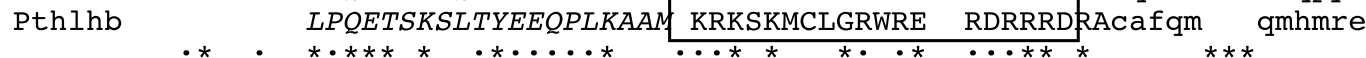
$\begin{array}{ll}\text { Carboxy-terminus } \\ \text { PTHLH } & \text { gdhlsdtsttsleldsr }\end{array}$ Pthlha c
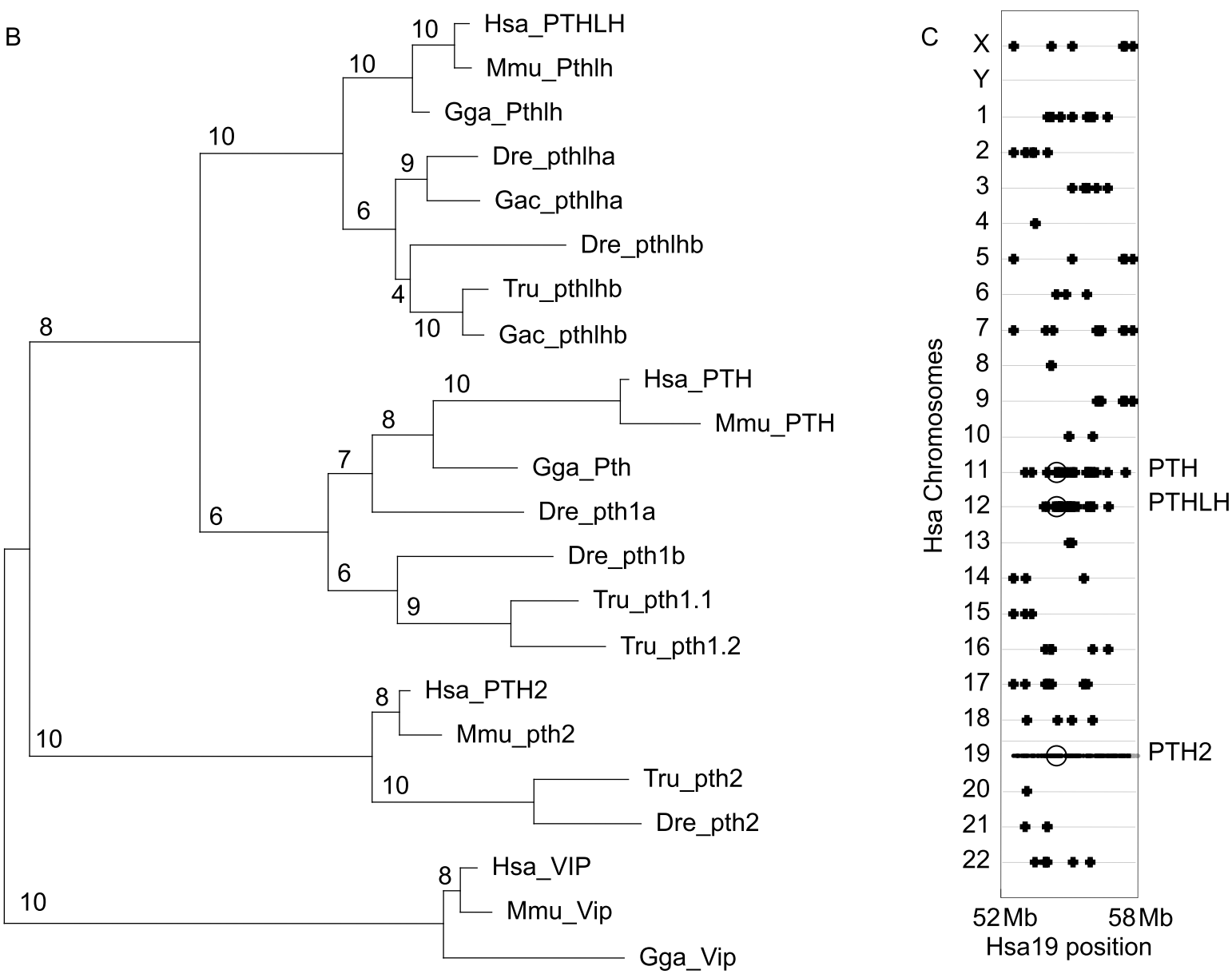
long bones in mutants slowly ossify from the outside in but retain a cartilaginous center, which may be due to high concentrations of Pthlh in the center of the cartilaginous bone mold (Weir et al. 1996). These results show that Pthlh plays an essential role in the development of the endochondral skeleton by modulating the timing and extent of chondrocyte differentiation and being tightly regulated by several factors such as Indian hedgehog (Ihh) and Sox9.

Pthlh acts through a reciprocal interaction with Ihh, at least in rodents (Karaplis et al. 1994, Vortkamp et al. 1996). Although ihh genes are expressed in zebrafish skeletogenesis (Avaron et al. 2006), the pthlh/ihh mechanism remains poorly understood in fish skeletogenesis (Provot \& Schipani 2005). Similarly, although zebrafish sox9 is required for chondrogenesis, a sox $9 /$ pthlh interaction is unknown (Yan et al. 2002, 2005).

Thus, because little is known about the roles of Pthlh in nonmammalian vertebrates, we isolated zebrafish pthlh genes to evaluate two hypotheses: 1) zebrafish pthlh is functionally conserved compared with human PTHLH and 2) similar to human PTHLH, zebrafish pthlh is expressed and tightly regulated during development. We found two co-orthologs of human PTHLH, as with several other genes that regulate craniofacial skeletogenesis, including $b m p 2$, $\operatorname{run} x 2$, sox9, and ihh, all of which arose during a genome duplication event in the teleost lineage, the teleost genome duplication (TGD; Amores et al. 1998, Postlethwait et al. 2000, Jaillon et al. 2004, Taylor \& Raes 2004, Nakatani et al. 2007). Duplicated genes derived from this genome duplication often partitioned ancient subfunctions between the co-orthologs (Force et al. 1999, Postlethwait 2007), which may help facilitate dissection of these pathways, including the regulatory mechanisms governing reciprocal Pthlh signaling between chondrogenic and osteogenic developmental programs (Yan et al. 2005, Flores et al. 2006). We show here that Sox9 upregulates Pthlh, which downregulates Runx2 during craniofacial and odontogenic development.

\section{Materials and Methods}

Identification of $g D N A$ regions encoding putative zebrafish pthlh sequences

A cDNA encoding a partial sequence of pthlh from the puffer fish Sphoeroides nephelus (accession DQ023267) was used as a probe to search the zebrafish genome website (http://134.174.23.160/HumanblastZebrafish/) for homologous sequences. Two sequences, pthlha (NM_001024627) on chromosome 4 and pthlhb (NM_001043324) on chromosome 25, were identified by BLAST analyses that showed the greatest sequence similarity to puffer fish and human PTHLH genes (PTHLH, accession M57293 and M32740).

Zebrafish (WT strain AB) were reared, anesthetized, and killed according to established IACUC-approved protocols (Westerfield 2000). We use the ZFIN guide for gene and protein nomenclature (http://zfin.org/zf_info/nomen.html). For example, genes for zebrafish, human, and mouse are designated, respectively, pthlha, PTHLH, and Pthlh and proteins Pthlh, PTHLH, and Pthlh. Although the official symbol is PTHLH, some of the aliases include PTHrP, HHM, Pthlp, PTH-L, and PLP (Abbink \& Flik 2007, Pinheiro et al. 2010).

\section{Isolation of zebrafish pthlh cDNAs}

For pthlha, $5^{\prime}$-RACE was performed on an adult zebrafish head cDNA library constructed in $\lambda$ ZAP (Stratagene) using platinum Taq DNA polymerase (Invitrogen) with the primers SK (5'-CCGCTCTAGAACTAGTGGATC) and reverse (REV) pthlha-1 (5'-CCTCCGGATGCCCTTCATCATCAT). Nested PCR was performed with SK and Rev pthlha-2 (5'-GATTTGTGGGTCTCCTGCTGCGG). Amplicons were gel purified, then ligated to pGEM-Teasy (Promega), and called pthlha-5'-RACE/pGEMT. 3'-RACE was performed with platinum Taq DNA polymerase, T7 primer (5'-TAATACGACTCACTATAGG), and primer

Figure 1 Phylogenetic and conserved synteny analysis. (A) Human (M57293 and M32740) and zebrafish Pthlha (DQ022615) and Pthlhb (DQ022616) amino acid sequences aligned (upper case) as described (Bhattacharya et al. 2011). The first residue of human PTHLH is designated as +1 (underlined). The signal sequence and 'pro' peptides (residues to the left of +1 ) are bold. The Pthlh sequence alignments revealed a 41 amino acid gap of low sequence homology plus conserved regions including the $\mathrm{N}$-terminus $(1-34$; 34 th residue is underlined), nuclear localization sequence (boxed) containing the RNA-binding domain, and mid-region (uppercase italics). Because the zebrafish genome contains two Pthlh orthologs that differ in their conservation in these regions, they are useful natural variants to evaluate the roles different domains play during chondrogenic and osteogenic development. *Identical residues: conservative substitutions (Flanagan et al. 2000). (B) Phylogenetic analysis by maximum likelihood, maximum parsimony, and neighbor joining methods agreed in topology (maximum likelihood shown using JTT substitution model, four substitution rate categories, http://atgc.lirmm.fr/phyml/ (Guindon et al. 2005). Bootstrap values are from ten replicates. Analyses suggest that PTH and Pthlh are sister groups and that PTH2 (alias TIP39) is the sister group to this super-clade. PTH, Pthlh, and PTH2 thus form a superfamily, whereas secretin falls as a sister group to this larger superfamily. (C) Dotplots mapping genes on Hsa19 from 52 to $58 \mathrm{Mb}$ displayed along the horizontal axis to their orthologs on the other human chromosomes displayed as a dot on the proper chromosome along the vertical axis directly above each $H$ sa 19 gene. Circles indicate the locations of PTH2 on Hsa19 and its paralogs PTH and PTHLH on human chromosomes Hsa11 and Hsa12 respectively. The PTH2 chromosome segment has paralogs mainly on Hsa11 and Hsa12. Tetrapods: chicken (Gallus gallus), Gga_Pth_M36522; human, Hsa_PTH_NM_000315; mouse, Mmu_Pth_NM_020623, Gga_Pthlh_X52131, Hsa_PTHLH_03580, Mmu_Pthlh_M60056,

Hsa_PTH2_NP_848544, Mmu_PTH2_NP_444486, Gga_Vip_AAA87896, Mmu_Vip_BAB31301, VIP_Hsa_NP_003372; Fish: zebrafish (Danio rerio), Dre_PTH1a_NM_212950, Dre_PTH1b_NM_212949, fugu Tru_pth1.1 (Danks et al. 2003), Tru_pth1.2_AAQ73561; Tru_pthlhb_AJ249391; stickleback (Gasterosteus aculeatus), Gac_pthlhb_ENSGACG00000004317, Gac_pthlha_ENSGACT00000000765, pthlha_Dre_NP_001019798, pthlhb_Dre_NP_001036789, Tru_pth2_T004305 Scaffold_4305, Dre_pth2_NP_991140. 
forward (FOR) pthlha (5'-ACCCACGCTCAGCTGATGCAT). Nested PCR was performed with T7 and primer For pthlha-3 (5'-GATAAAGGCCGAACGCTGCAG). Amplicons were gel purified, then ligated to pGEMT, and called pthlha3'-RACE/pGEMT. Zebrafish pthlha-5'-RACE/pGEMT and pthlha-3'-RACE/pGEMT were used to transform Escherichia coli TOP 10 cells (Invitrogen). Plasmids containing pththa $5^{\prime}$-RACE and $3^{\prime}$-RACE cDNAs in pGEMT were sequenced according to manufacturer protocols (ABI, Perkin-Elmer Corp., Foster City, CA, USA).

For pthlhb, total RNA was reverse transcribed with Superscript II reverse transcriptase (Invitrogen) and Rev pthlhb-1 (5'-CTTTTATCACTCTCTCATGTGCAT). To isolate the cDNA encoding the $5^{\prime}$-nucleotides of pthlhb, a 1:100 dilution of the reverse transcriptase reaction was amplified with Rev pthlhb-3 (5'-CATCTTACTCTTCCTCTTCATG) and For pthlhb-5 (5'-ATGTTAAGGCACTGGGGCTT). A nested amplification used the following primers: Rev pthlhb-4 (5'-TGGCTGCTCTTCATATGTCAG) and For pthlhb-5. To identify the $3^{\prime}$-RACE cDNAs encoding pthlhb, total zebrafish RNA was reverse transcribed using Superscript II reverse transcriptase and an oligo-dT anchor primer (Bhattacharya et al. 2011). The first strand was then amplified by PCR using the primer For pthlhb-1 (5'-GTCACGCTCAGATGATGCAC) and AUAP primer (5'-GGCCACGCGTCGACTAGTAC; Invitrogen). A nested amplification used the following primers: For pthlhb-2 (5'-ACAGGAGCCGCTCTCTGCAT) and an adapter AUAP primer. 3'-RACE cDNAs encoding pthlhb were isolated, ligated to pGEM-Teasy, and sequenced as described earlier.

Determination of intron/exon boundaries, sequence alignments, and phylogenetic analyses

The intron/exon structure of the zebrafish pthlh genes was determined using (http://www.searchlauncher.bcm.tmc. edu/seq-search/gene-search.html), location of RNA splice sites was determined using (http://www.fruitfly.org/seq_ tools/splice.html), and signal sequence was predicted using (http://www.cbs.dtu.dk/services/SignalP/). Sequence alignments and phylogenetic analyses were performed as described (Guindon et al. 2005, Bhattacharya et al. 2011).

\section{Zebrafish sox $9 a^{-}$and sox $9 b^{-}$mutants}

sox 9 single mutants $\left(\operatorname{sox} 9 a^{-}\right.$and $\left.\operatorname{sox} 9 b^{-}\right)$and double mutant $\left(\operatorname{sox} 9 a^{-} / \operatorname{sox} 9 b^{-}\right.$; Yan et al. 2002, 2005) were used to evaluate the expression of pthlha and pthlhb during development.

\section{Zebrafish pthlh antisense morpholino oligonucleotide injection}

Antisense morpholino oligonucleotides (MOs) were obtained (http://www.gene-tools.com) and injected in one- to twocell zebrafish embryos as described (Draper et al. 2001). The zebrafish pthlha splice-blocking MO (e2i2 (exon-2, intron-2)
5'-CACAGACACTTACATTCGGCTGCTG, which blocks the exon-2 donor site, Fig. 6A) or the pthlhb splice-blocking MO (i2e3 (intron-2, exon-3) 5'-CTGAACGCCGTCTGGAACATAGACA, which blocks the exon-3 acceptor site, Fig. $6 \mathrm{~B} ; 1 \cdot 0-2 \cdot 0 \mathrm{nl}$ of a $3.0 \mathrm{mg} / \mathrm{ml} \mathrm{MO}$ stock), was injected into about 500 embryos each session for at least three independent sessions to assess phenotypes compared to noninjected embryos. Controls were of two types: 1) the injection of control MO (TTTTTGCTCATACAGGTGGCCTGAG, which represents the sense $M O$ for zebrafish egr2b, NM_183341) and which gave no effect, and 2) the co-injection of pthlha or pthlhb MO along with tp53 (NM_131327) MO (GCGCCATTGCTTTGCAAGAATTG) at $2 \cdot 0 \mathrm{mg} / \mathrm{ml}$, which follows the recommended procedure to ensure that $\mathrm{MO}$-induced phenotypes are not caused by nonspecific cell death due to MO toxicity (Robu et al. 2007, Eisen \& Smith 2008). MO-injected embryos were allowed to develop to 24,48 , and $72 \mathrm{~h}$ post-fertilization (hpf) before whole-mount in situ hybridization, examining at least 50 animals per time point (Bhattacharya et al. 2011). To evaluate disruption of pthlha or pthlhb $\mathrm{mRNA}$, RT-PCR was performed on noninjected controls and pooled MO-injected embryos using either pthlha primers (For +120, 5'-AACTTTGAGGAGACTGGCGCT; Rev-745, 5'-AACTCCTGCAGTGATCGAGCT; Fig. 6A) or pthlhb primers (For-in, 5'-CGCTGTGGTGCCCCCTGATAA; Rev-R, 5'-CTTTTATCACTCTCTCATGTGCA; Fig. 6B).

Whole-mount in situ hybridization and alcian blue/alizarin red staining

Zebrafish pthlha and pthlhb cRNA probes were produced by linearizing pthlha-5'-RACE/pGEMT with NotI and pthlhb$5^{\prime}$-RACE/pGEMT with NcoI and then transcribing the cDNA using the DIG RNA labeling kit (Roche Applied Science) using T7 RNA polymerase for pthlha and Sp6 for pthlhb probes. The whole-mount in situ hybridization on zebrafish embryos was performed as described previously (Yan et al. 2002, Bhattacharya et al. 2011). For cellular resolution, cryosections were subjected to in situ hybridization as described (Rodriguez-Mari et al. 2005). Alcian blue and alizarin red staining of cartilage and bone were performed as described (Walker \& Kimmel 2007).

\section{Results}

Identification of cDNAs encoding zebrafish pthlha and pthlhb

To identify zebrafish pthlh genes, we used the pthlh cDNA sequence of $S$. nephelus to search the zebrafish genome database. Two putative zebrafish gDNA regions were identified that showed sequence similarity to the human PTHLH transcript, which we call pthlha and pthlhb. To determine whether these putative genes are expressed, genespecific primers for zebrafish pthlha and pthlhb were designed 
to amplify products by RT-PCR using adult zebrafish total RNA. Based upon partial pthlha and pthlhb cDNA sequences, $5^{\prime}$ - and $3^{\prime}$-RACE reactions were performed to obtain the full-length cDNAs (accession: pthlha, DQ022615 and Ensembl, ENSDARG00000031737; pthlhb, DQ022616 and Ensembl, ENSDAR G00000071070).

Genomic regions with sequence identity to the cDNA sequences were analyzed to predict intron-exon boundaries. The organization of the zebrafish pthlha and pthlhb genes is simpler than that of the human PTHLH gene due to the lack of exons encoding additional C-terminal variants. In general, the gene structures for pthlha and pthlhb are organized similar to PTHLH in which the $5^{\prime}$-UTR is encoded on exon- 1 , exon-2 encodes the prepro sequence (presequence includes -40 through -7 for Pthlha and -32 through -7 for Pthlhb, and part of the prosequence, including -6 through $-3)$, and exon-3 encodes the rest of the prosequence $(-2$ and -1$)$ and the mature peptide (ENSDARG 00000031737 and ENSDARG00000071070). More specifically, Pthlha encompasses three exons where exon-1 encodes the $5^{\prime}$-UTR, exon-2 encodes the transcription start site and $\mathrm{N}$-terminus, and exon-3 encodes the midregion, NLS and RNA-binding region, and C-terminus regions of Pthlh (ENSDARG00000031737). Although Pthlha exon-1 encoded 219 nucleotides of $5^{\prime}$-UTR, the longest transcript encoding Pthlhb that we isolated lacked exon-1, contained exon-2 encoding 18 nucleotides of $5^{\prime}$-UTR as well as the transcription start site and $\mathrm{N}$-terminus, and exon-3 encoding a conserved mid-region but a poorly conserved NLS and C-terminus regions (ENSDARG00000071070).

\section{Structural analysis of zebrafish Pthlh polypeptides}

Similar to PTHLH, each zebrafish Pthlh protein contained a predicted signal sequence (Supplementary Table 1, see section on supplementary data given at the end of this article) and dibasic cleavage site (either RR or KR) immediately preceding the first amino acid residue of the predicted mature peptide (Fig. 1A). The predicted mature Pthlha and Pthlhb polypeptide hormones are 158 and 130 amino acids long respectively (Fig. 1A). Tblastx pairwise alignments showed that Pthlha is more similar to PTHLH (54\% identity) than Pthlhb (35\%), while the two zebrafish Pthlh proteins showed 57\% identity to each other (Fig. 1A). Regions that showed the greatest similarity to PTHLH and conservation with other Pthlh ligands include the Pthlh(1-34) N-terminus of the mature peptide, while the mid-region (Wu et al. 1996) and NLS/RNA-binding domain (Henderson et al. 1995, Nguyen \& Karaplis 1998) showed less conservation. Zebrafish Pthlh showed several N-terminal residue changes (A1S, H5A/V, and F23W) that are similar to residues in human PTH (hPTH), suggesting that Pthlh may function similar to hPTH for calcium regulation, while the Pthlhb mid-region showed the least conservation with a gap of 49 residues and a significantly degraded NLS sequence
(Gardella \& Jüppner 2000), which may suggest a lack of conservation for nuclear functions.

\section{Phylogenetic relationships among PTH, Pthlh,} and PTH2 peptides

A key question is the origin of the zebrafish pthlh gene duplicates. Using PhyML (Guindon et al. 2005), we analyzed phylogenetic relationships of Pthlh, PTH, and PTH2 from teleosts (zebrafish, stickleback, and puffer fish) and tetrapods, using VIP, which represents the next most closely related gene clade, as outgroup (Bhattacharya et al. 2011). While the Pthlh clade had highly significant bootstrap support (ten of ten replicates, Fig. 1B), the PTH clade was strong but not quite as robust (six of ten replicates). The Pthlh and PTH clades clearly grouped significantly as sister groups (eight of ten replicates), with the PTH2 (alias TIP39) clade grouping basal to the PTH-Pthlh supergroup, using VIP as outgroup. Within each Pthlh, PTH, and PTH2 clade, sequences followed the accepted phylogenetic relationships of the species themselves, from basal to derived. Teleost Pthlha and Pthlhb sequences fell as duplicates arising after the divergence of the teleost and tetrapod lineages, consistent with the interpretation that they are co-orthologs of human PTHLH, having arisen in the TGD event.

\section{Genomic origin of the Pthlh family}

The phylogenetic analysis confirmed that Pthlh, PTH, and PTH2 form a tight gene family. To further understand the historical relationships of these genes, we used our automated engine for the analysis of conserved syntenies beginning with PTH2 (Catchen et al. 2009). For every gene in a $6 \mathrm{Mb}$ region centered on PTH2 in human chromosome 19 (Hsa19), the engine searched for paralogs on other human chromosomes and placed a dot on the chromosome above the Hsa19 gene. Open circles in Fig. 1C locate PTH2 on Hsa19 and its paralogs $P T H$ and $P T H L H$ on Hsa11 and Hsa12 respectively. Results showed that genes neighboring PTH2 have paralogs on many human chromosomes, but Hsa11 and Hsa12 are the major paralogons for the region of Hsa19 containing PTH2; these two regions have paralogs of 27 and $23 \mathrm{Hsa} 19$ genes respectively (Fig. 1C). Hsa7 and Hsa1 have 14 and 15 paralogs each and fewer other chromosomes. These results would be expected if an original chromosome segment containing a single PTH/PTHLH gene experienced two rounds of duplication to make four chromosome segments, three of which today are parts of human chromosomes 11, 12, and 19 containing PTHLH, PTH, and PTH2, respectively, and the fourth is part of either Hsa1 or Hsa7, but this fourth segment has lost its PTHLH-related gene, the most common fate of duplicated genes. We conclude that the vertebrate PTHLH, $P T H$, and PTH 2 genes arose in the two rounds of genome duplication that occurred at the base of the vertebrate radiation (Garcia-Fernandez \& Holland 1994, Dehal \& Boore 2005). 
Syntenic relationships of zebrafish pthlh genes

Inferring a gene's origins requires the investigation of its conserved syntenies. Fig. 2A, B, C, D and E compares genomic regions surrounding the two zebrafish pthlh genes to the human genome, while Fig. $2 \mathrm{~F}$ and $\mathrm{G}$ compares, reciprocally, the genomic region surrounding the human PTHLH gene to the two zebrafish chromosomes. Zebrafish pthlha and pthlhb reside at the left ends of linkage groups (LGs) 4 and 25 respectively (Fig. 2A and E). Figure $2 \mathrm{~B}$ shows an enlargement of the marked region on LG25 in Fig. 2A. Four genes immediately flanking pthlhb (Fig. 2B) have human orthologs, as judged by best reciprocal BLAST analysis (Wall et al. 2003), on Hsa12 (Fig. 2C), and orthologs of four genes immediately to the left of pthlha (Fig. 2D) are also located on Hsa12 (Fig. 2C). Reciprocally, of the 20 annotated genes surrounding PTHLH on Hsa12 (Fig. 2G, an enlargement of the bar indicates the position of PTHLH in Fig. 2C), all except one has orthologs on zebrafish LG4 or LG25
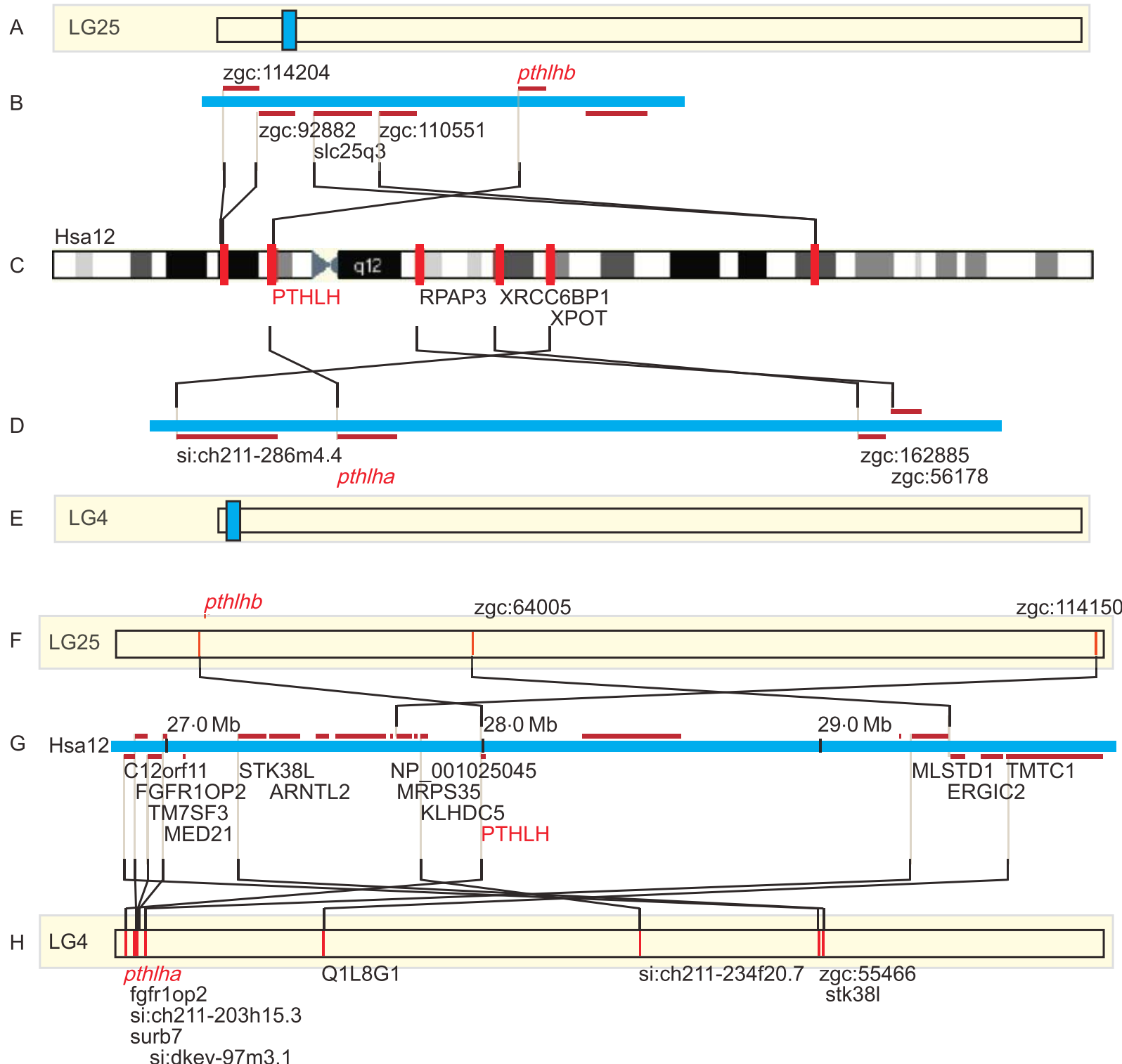

Figure 2 Conserved syntenies for zebrafish pth/ha and pth/hb genes. (A, E, F and H) Represent entire zebrafish chromosomes (Zv7). ( $B$ and $D$ ) Show all annotated genes (red horizontal lines) in the regions of the zebrafish chromosomes indicated in blue in $A$ and $E$. (C) Shows an ideogram of human chromosome 12, with the locations of human orthologs of zebrafish genes shown as red bars. Lines link orthologs between the two species. (G) Shows the region of human chromosome $12 \mathrm{p} 11.2$ with all annotated genes indicated in red horizontal lines. Annotated sequences for which no zebrafish ortholog is called are in gray. Black lines link human genes to zebrafish orthologs. The ortholog of ARNTL2 is on LG18. 
(Fig. 2F and $\mathrm{H}$ ). The most parsimonious explanation for these data is that the last common ancestor of zebrafish and humans had a single chromosome region with this gene set, that the chromosome segment duplicated in the zebrafish lineage, and that the two duplicated regions evolved into the current genomic arrangements by gene loss and inversions. Because pthlha and pthlhb are parts of this duplicated chromosome region, we conclude that they are co-orthologs of the human PTHLH gene arising in the TGD event.

\section{Early developmental expression of zebrafish Pthlh co-orthologs}

Key insights into a gene's functions come from its pattern of expression. RT-PCR of one cell, 5, 10, 24, 48, and $72 \mathrm{hpf}$ embryos detected transcripts of pthlha and pthlhb at all stages (Supplementary Fig. 1, see section on supplementary data given at the end of this article). These results showed that pthlha and pthlhb are maternally expressed but at levels that are not clearly detected by in situ hybridization (Fig. 3A and B). At 12, 24, and $48 \mathrm{hpf}$, both pthlha and pthlhb showed expression in the region of presumptive branchial arch-4 (Fig. 3C, D, E, F, G and H). At 72 hpf, pthlha was expressed in otic vesicles (ov), superoptic cartilages (sopc), and pancreas (pan) (Fig. 3I); in tooth-forming cells (tee; Philbrick et al. (1998)); and in scattered cells of the retina similar to the distribution of amacrine cells (eye (e); Fig. 3K; Yokoi et al. 2009; Fig. 3M). These expression domains are similar to those of Pthlh in developing mouse embryos (Lee et al. 1995, Philbrick et al. 1996). Surprisingly, zebrafish embryos did not appear to express pthlh genes in keratinocytes of the skin (Wysolmerski et al. 1994) or scales or fins (Trivett et al. 1999). At $72 \mathrm{hpf}$, pthlhb was expressed in a triangular patch of 20-30 mesenchymal cells of unknown identity near the presumptive thymus (Figs 3J, L and $4 \mathrm{H}$ ) and in spinal neuromasts (Fig. 3N), where it may be associated with the formation of the caudal neurosecretory system (urophysis; Ingleton et al. 2002). The ceratohyal and opercular provided useful elements for position due to their location and size (Albertson et al. 2005).

Pthlh co-ortholog expression during tissue patterning and ossification

In situ hybridization studies on cryosections revealed the expression pattern of pthlha and pthlhb in the developing craniofacial skeleton in single-cell detail. In histological sections, pthlha expression appeared robustly throughout the brain (Fig. 4A, $53 \mathrm{hpf}$ ), the cartilage core of branchial arches 1-4 (Fig. 4C, 3 dpf), pancreatic endocrine cells (Fig. 4E, $3 \mathrm{dpf}$; Shor et al. 2006), retinal amacrine cells within the inner nuclear layer and ganglionic layer of the retina (Fig. 4I, $6 \mathrm{dpf}$; Yokoi et al. 2009), teeth (Fig. 4K, $6 \mathrm{dpf}$ ), spinal cord (Fig. 4M, $6 \mathrm{dpf}$ ), and in cartilages, including the perichondrium and in hypertrophic chondrocytes, for example, the ceratohyal (Fig. 4G, $6 \mathrm{dpf}$ ). In contrast to pthlha, expression of pthlhb was not generally associated with the development of brain, spinal cord, pancreas, or teeth but showed weak expression in

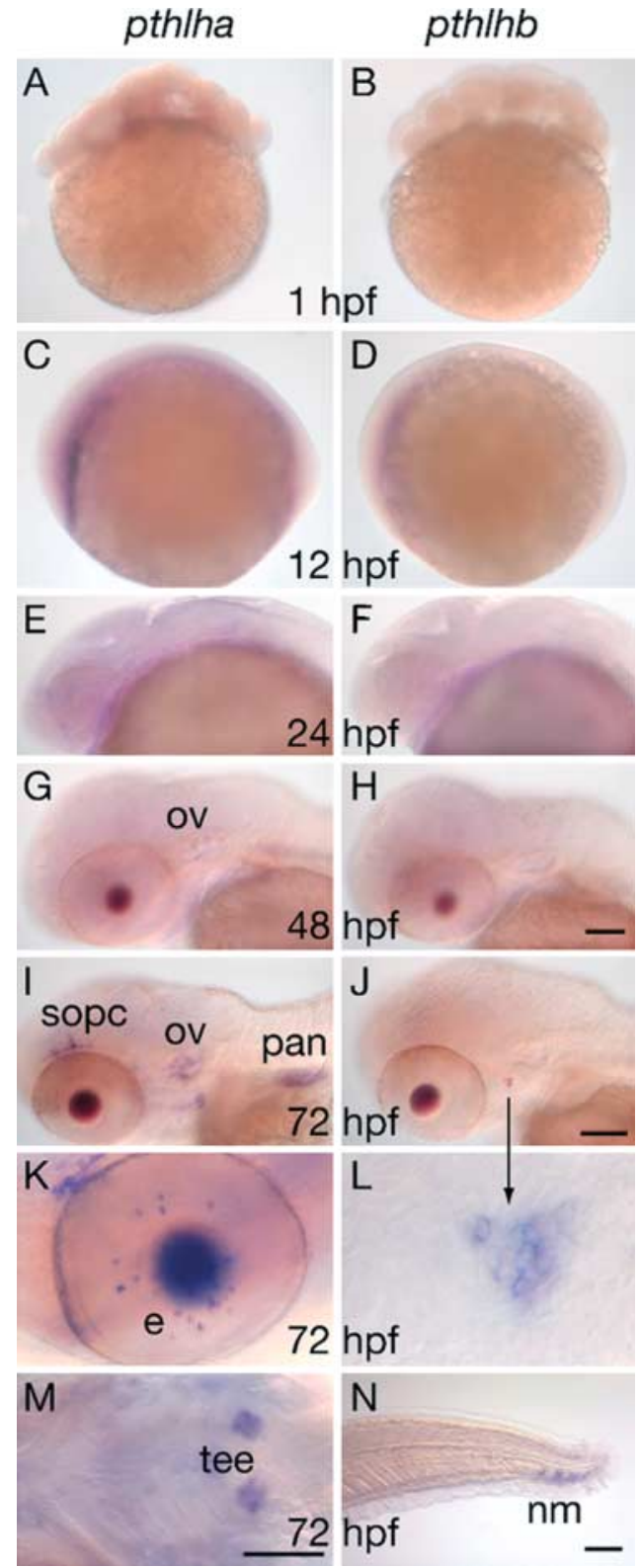

Figure 3 Tissue-specific expression of zebrafish $p t h / h$ co-orthologs. Expression of pthlha and pth/hb assessed by whole-mount in situ hybridization (Pthlha panels A, C, E, G, I, K and M; Pthlhb panels B, D, F, H, J, L and N) assessed at 1, 12, 24, 48, and 72 hpf. Panels A and $B$ show very low level of expression at the embryo-yolk interface for both $p t h / h$ mRNAs. The pthlha gene was expressed in the otic vesicle (ov, panels G and I); super optic cartilages (sopc), pancreas (pan, panel I), retina (e, panel K), and teeth (tee, panel M). The $p t h / h b$ gene was expressed in the pharyngeal arch region (panels J and $\mathrm{L}$, arrow) and urophysis (neuromasts) of the tail (nm, panel N). Panels A, B, C, D, E, F, G, H, I and J, lateral view, anterior toward the left. All scale bars, $100 \mu \mathrm{m}$. 
the eye (Fig. 4B, $53 \mathrm{hpf}$ ), hypertrophic chondrocytes of the hyosymplectic and opercular (Fig. 4D and F, 4 dpf), and in the proximity of the thymus (Fig. $4 \mathrm{H}, 4 \mathrm{dpf}$; rag1-labeled cRNA probe, control).

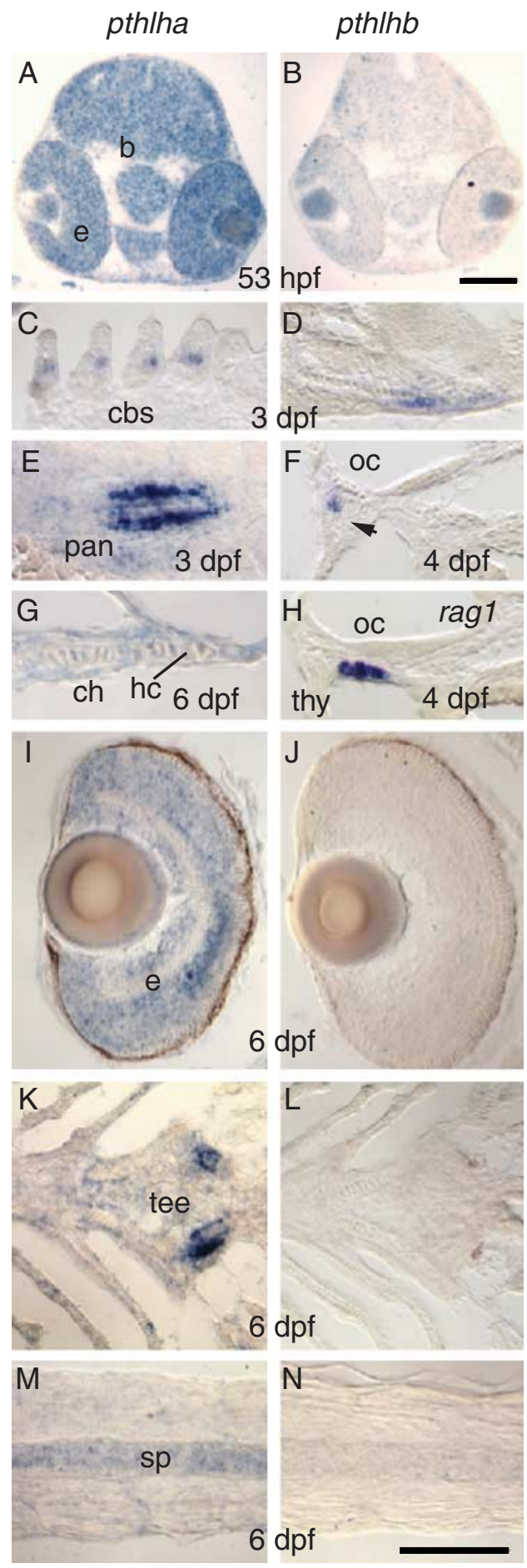

The region around the pharynx gives rise to not only elements of the craniofacial skeleton but also to the thymus, thyroid, and in terrestrial vertebrates to parathyroid glands, so we asked whether the pharyngeal pthlhb domain included any of these organs. The pthlhb expression domain in the pharyngeal arch region is unlikely to be the thyroid because in fish embryos the thyroid develops as an unpaired structure in the midline (Rohr \& Concha 2000, Alt et al. 2006) rather than as a paired organ in the arches like the pthlhb expression domain (Figs 3J and L, 4H).

To ask whether the pharyngeal domain of pthlhb-expressing cells is related to the thymus, we compared the expression pattern of pthlhb to that of the thymus marker rag1 (Willett et al. 1997). Results showed that the rag1 domain was more ventrally and centrally located than the pthlhb domain (Figs 4F, $\mathrm{H}$ and $5 \mathrm{E}, \mathrm{I}, \mathrm{M}, \mathrm{Q})$. Sections showed that, while the rag1 domain was in the endoderm (Fig. 4H), the pthlhb domain was expressed at the posterior border of the hyosymplectic and opercular (Fig. 4D and F) ruling out the possibility that pthlhb expression labels thymus cells.

Fish lack anatomically distinct parathyroid glands, which is a major difference between the teleost and tetrapod endocrine systems (Wendelaar Bonga \& Pang 1991). Furthermore, in zebrafish, pth 1 and pth 2 are not expressed in cells likely to correspond to a gland equivalent to a pharyngeal-derived parathyroid but are expressed in early sites of skeletal calcification (Hanaoka et al. 2004, Hogan et al. 2004). Thus, although the pthlhb-positive domain is approximately in the position expected for parathyroid gland cells, it is unlikely to represent the parathyroid.

\section{Regulatory interactions of Sox9 and Pthlh}

Because zebrafish sox9 genes are essential for pharyngeal arch development (Yan et al. 2005), we wondered whether this transcription factor is necessary for the development of pthlh-expressing cells of the arches. Zebrafish has two Sox9 co-orthologs, $\operatorname{sox} 9 a$ and $\operatorname{sox} 9 b$, that are derived from the TGD and are involved in different aspects of arch development (Chiang et al. 2001, Yan et al. 2005). To discern whether zebrafish $\operatorname{sox} 9 a$ or sox $9 b$ regulates pthlh-expressing cells, we evaluated pthlh gene expression in single mutants lacking function of one or the other sox 9 co-ortholog and in double mutants. Results showed that pthlha expression was diminished

Figure 4 Histological analysis of tissue-specific expression of zebrafish pth/h co-orthologs. Expression of pthlha and pth/hb transcripts in sections of zebrafish embryos (pth/ha panels A, C, E, G, I, K and M; pth/hb panels B, D, F, H, J, L and N) at 53 hpf, 3, 4, and $6 \mathrm{dpf}$. Expression of pth/ha was robust in the brain (b) and retina $(\mathrm{e}, \mathrm{A})$, ceratobranchial arch cartilages (cbs, C), pancreas endocrine cells (pan, E), hypertrophic chondrocytes (hc) of the ceratohyal cartilage (ch, G), eye (e, A, I), teeth (tee, K), and spinalcord (sp, M). Expression of pthlhb was slight in the eye (retina, B), chondrocytes at the posterior border of the hyosymplectic and opercular (Fig. 4D and $\mathrm{F}$ respectively), and the thymus region (thm, $\mathrm{H}$; rag1-labeled cRNA probe, control); oc, opercular chondrocytes ( $\mathrm{F}$ and $\mathrm{H})$. All scale bars, $100 \mu \mathrm{m}$. 


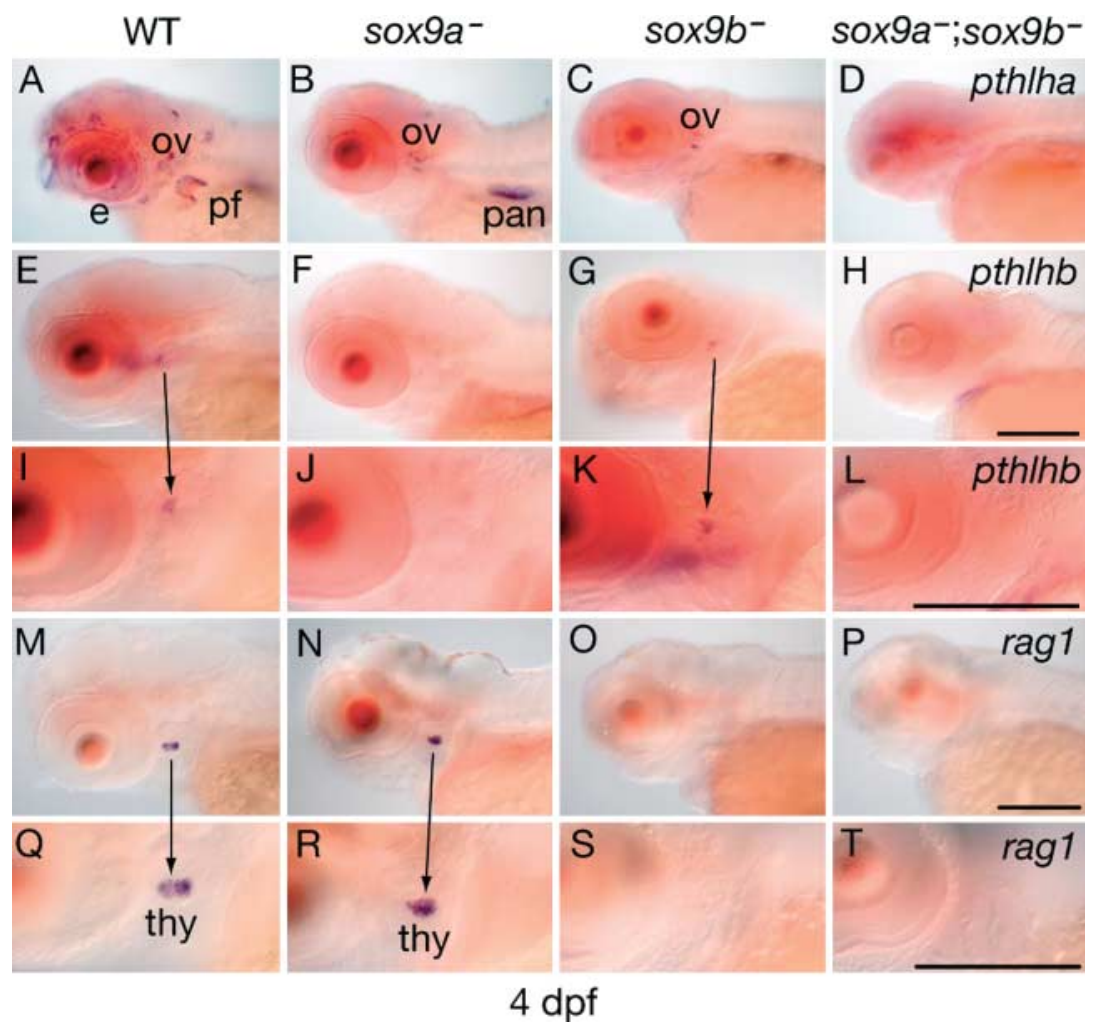

Figure 5 pth/h expression in sox 9 mutants. Expression of pth/ha, pth/hb, and rag1 in WT,

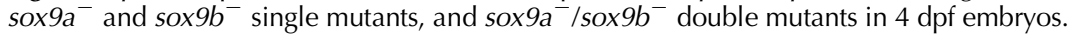
pthlha expression (A) was diminished in the pharyngeal arches, superoptic cartilages, and pectoral fin buds of sox9a (B) and sox $9 b$ mutants (C). In double mutants, most pthlha expression was eliminated (D). We conclude that sox9a regulates pthlha expression in many craniofacial cartilages and in the pectoral fin ( $\operatorname{sox} 9 a \rightarrow$ pthlha in chondrocytes) and that $p$ th/ha is downstream of sox9a in some tissues and downstream of sox9b in other tissues. pthlhb (and not $p$ th/ha) showed expression in the a distinctive domain about 20 cells in the pharyngeal arch region $(\mathrm{E}, \mathrm{I}$ and $\mathrm{G}, \mathrm{K})$. pth/hb expression in that pharyngeal arch region is regulated by sox $9 \mathrm{a}$ $(\mathrm{F}, \mathrm{J})$ while rag1 expression in thymus is regulated by $\operatorname{sox} 9 b(\mathrm{O}, \mathrm{S})$. The arrows show the hybridized region in a more amplified image below. Thus for example, the triangular region on panel $\mathrm{E}$ is shown in panel $\mathrm{I}$ at a greater magnification. Similarly the rag1 expression in panel $\mathrm{M}$ is shown in panel $\mathrm{Q}$ at a greater magnification. e, retina; ov, otic vesicle; pan, pancreas; pf, pectoral fin; and thm, thymus. Anterior towards the left. Scale bars, $100 \mu \mathrm{m}$.

in the pharyngeal arches, sopc, and pectoral fin buds of $\operatorname{sox} 9 a$ mutants (Fig. 5A and B). We conclude that sox $9 a$ regulates pthlha expression in many craniofacial cartilages and in the pectoral fin (sox $9 a \rightarrow$ pthlha in chondrocytes). This is consistent with the known role of $\operatorname{sox} 9 a$ in the development of osteochondroprogenitor cells, including the cartilaginous pharyngeal skeleton (Akiyama et al. 2005, Yan et al. 2005). By contrast, the expression of pthlha in the pancreas was unaffected (Fig. 5B). In sox $9 b$ mutants (Fig. 5C), pthlha expression was also reduced in the arches and, in addition, was reduced in the pancreas, a known target of Sox 9 action (Seymour et al. 2008). We conclude that $s o x 9 b$ rather than $s o x 9 a$ regulates pancreatic expression of pthlha (sox9b $\rightarrow$ pthlha in pancreas). In double mutants, most pthlha expression was eliminated (Fig. 5D). Thus, we conclude that pthlha is downstream of sox $9 a$ in some tissues and downstream of $s o x 9 b$ in other tissues.
Sox9 activity is also necessary for embryonic expression of pthlhb: the expression of pthlhb disappeared in sox $9 a$ but not in sox $9 b$ mutants (Fig. 5F, J, G and $\mathrm{K}$ respectively). We conclude that $\operatorname{sox} 9 a$ but not $\operatorname{sox} 9 b$ function is required for expression of the pthlhb domain in the pharyngeal arches and/or for the development of pthlhb-expressing cell types $(\operatorname{sox} 9 a \rightarrow$ pthlhb $)$. The thymus, as defined by rag 1 expression, was controlled by $s o x 9 b$ but not $\operatorname{sox} 9 a$ (Fig. $5 \mathrm{O}, \mathrm{S}, \mathrm{N}$ and $\mathrm{R}$ respectively). The finding that $\operatorname{sox} 9 a$ regulates the pthlhbpositive cells but that sox $9 b$ regulates the rag1-expressing cells (Fig. 5M, N, Q and R) is consistent with the interpretation that the pthlhb-expressing cells are a different cell type from the rag1-expressing cells (Fig. 5E, I, G and $\mathrm{K})$. Overall, these results show that $\operatorname{sox} 9 a$ stimulates expression of pthlha and pthlhb $(\operatorname{sox} 9 a \rightarrow$ pthlha and pthlhb) in chondrocytes. 
The sox9 mutants help to rule out possible identities of the small triangular patch of pthlhb-expressing cells. In sox9a mutants, the opercular bone develops normally (Yan et al. 2005) but the triangular patch of pthlhb-expressing cells is gone. By contrast, in sox $9 b$ mutants, the opercular is greatly reduced but the pthlhb domain is normal. This shows that pthlhb is not expressed in the opercular.
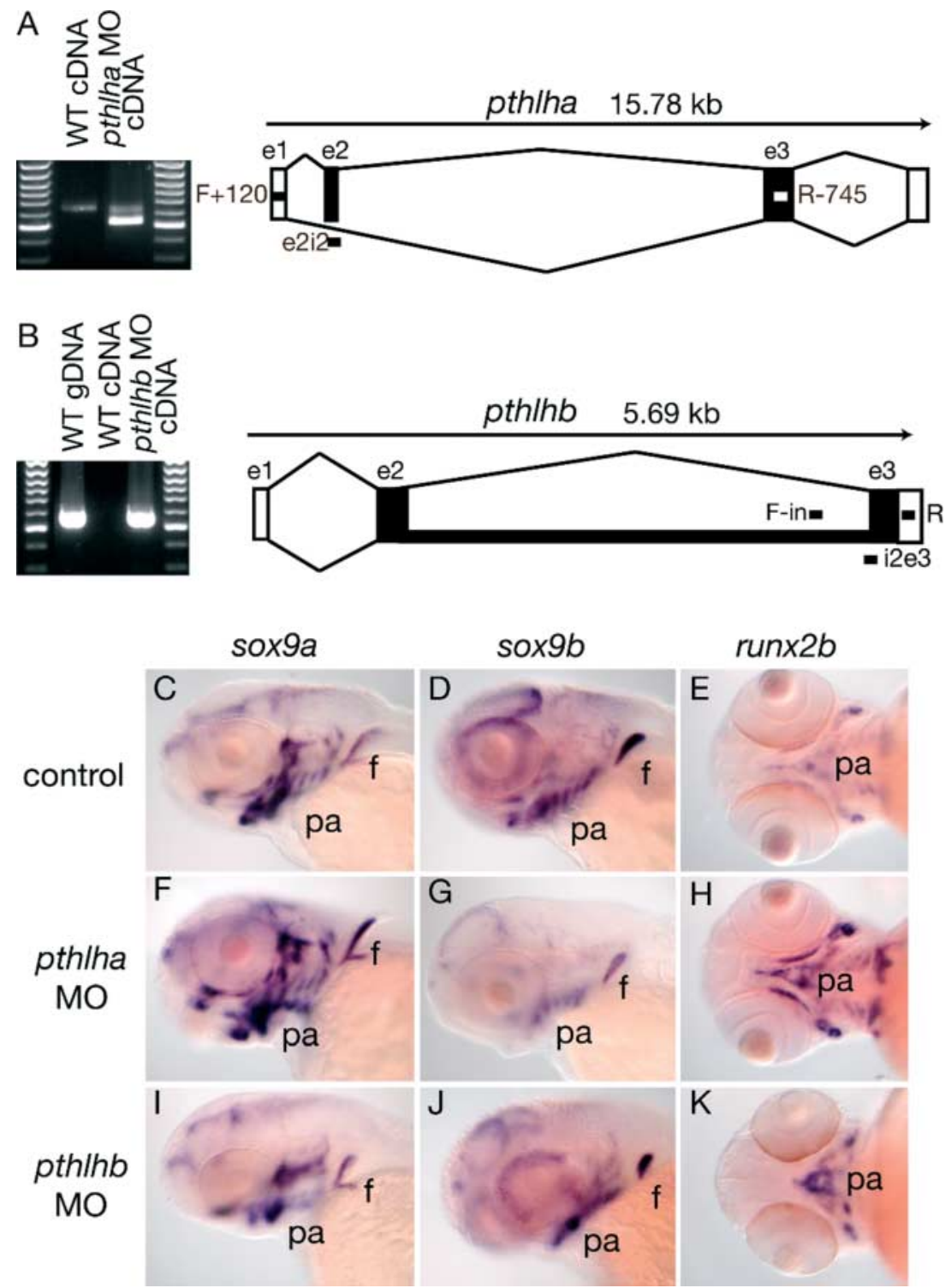

Figure 6 Morpholino knockdown of pth/ha and pth/hb. (A) pth/ha; left, pth/ha amplicon size difference between CDNAs isolated from WT and MO-treated embryos, right, schematic indicating exons 1-3 (boxes), introns (lines), primers (forward, F+120 and reverse, $R-745$ ), and splice-blocking $\mathrm{MO}(\mathrm{e} 2 \mathrm{i} 2)$. Compared with $\mathrm{WT}$, the pthlha morphant showed a significantly decreased amplicon size due to the elimination of exon-2 from the pre-mRNA. (B) pth/hb; left, pth/hb amplicon size difference between WT and MO-treated embryos. Right, schematic indicating the location of exons 1-3, primers (forward, F-in and reverse, R) and splice-blocking MO (i2e3). Compared with WT gDNA, the pthlhb morphant showed a similar amplicon size indicating the lack of intron excision in the mRNA whereas WT cDNA yielded no amplicon. (C, D, E, F, G, H, I, J and K) Regulation of sox9 expression in pth/ha knockdown embryos (C, F and I) and pth/hb knockdown embryos (D, G and J) at $3 \mathrm{dpf}$ queried by in situ hybridization for sox $9 a$, sox $9 b$, and runx $2 b$ as indicated in the figure. C, D, F, G, I and J, lateral views. E, H and K, ventral views with anterior to the left. $\mathrm{f}$, pectoral fin; pa, pharyngeal arches. 


\section{Evaluation of Pthlh knockdown phenotypes}

To understand the roles of pthlha and pthlhb in development, we knocked down activity using antisense MOs directed against splice donor and acceptor sites. To assess the efficacy of knockdown, we isolated total RNA from control or morpholino-injected embryos and performed RT-PCR using genomic DNA as control. The pthlha splice-blocking MOs were targeted to the splice donor site of intron-2 (MO-p1e2i2) and the splice acceptor site of intron-2 (MO-p1i2e3), which should delete exon-2 and thus remove the signal sequence and hence block secretion. To check whether the MOs did indeed delete exon-2, we amplified cDNA using primers in exon-1 and exon-3 (Fig. 6A). For pthlha, MO injection blocked the excision of intron-2 and caused the intron- 1 donor site to splice directly to the intron-2 acceptor site, thereby deleting exon-2 (Fig. 6A, left panel). Because the quantity of the exon-2-deleted transcript was greater than the quantity of normal transcript, we concluded that knockdown of pthlha was substantial. For pthlhb, spliceblocking MOs were designed for the splice acceptor site of intron-1 (MO-p2i1e2) and the splice acceptor site of intron-2 (MO-p2e2i2). Efficacy of knockdown was checked using primers in intron-2 and in the $3^{\prime}-\mathrm{UTR}$ (Fig. 6B). RT-PCR analysis showed no amplification from RNA extracted from normal controls, as expected if the mature mRNA lacks intron-2, the site of one of the primers. By contrast, results showed the substantial amplification of a band of $586 \mathrm{bp}$ from RNA isolated from MO-treated animals, as expected from an aberrant transcript that had failed to be spliced (Fig. 6B, left panel). We conclude that these MOs knocked down normal splicing of pthlhb, but they did not cause message degradation because whole-mount in situ hybridization experiments on pthlha and pthlhb MO-injected embryos at 24, 48, and $72 \mathrm{hpf}$ showed that neither the pthlha nor the pthlhb MO altered the level of pthlha and pthlhb transcripts (Supplementary Fig. 2, see section on supplementary data given at the end of this article), supporting the finding of altered transcript by RT-PCR (Fig. 6A and B).

To examine the role of zebrafish pthlh co-orthologs during skeletal development, we studied their epistatic relationships with the skeletal regulatory genes $\operatorname{sox} 9$ and $r u n \times 2 b$. MOs were injected into one-cell embryos and developing embryos were evaluated for the expression of $\operatorname{sox} 9 a$, sox $9 b$, and run $\times 2 b$ (Fig. 6C, D, E, F, G, H, I, J and K). Control animals injected with a standard control MO gave no phenotype different from uninjected embryos. To guard against phenotypes caused by nonspecific effects involving cell death, animals were injected simultaneously with pthlh MOs and a MO directed against p53 (Robu et al. 2007, Eisen \& Smith 2008). The phenotype of double knockdown animals was the same as the pthlh single knockdowns, so we conclude that the pthlh MOs do not cause off-target effects mediated by p53-related cell death. In normally developing $2 \mathrm{dpf}$ embryos, pthlha and sox9a expression overlaps in branchial arches and otic vesicles (Figs 3G, I and 4C, G) while sox $9 a$ is expressed in the forebrain, dorsal hindbrain, branchial arches, otic vesicles, pectoral girdle, and limb buds (Fig. 6C), and sox $9 b$ is expressed in the tectum, retina, dorsal hindbrain, otic vesicle, branchial arches, and pectoral fin bud (Fig. 6D), as described previously (Chiang et al. 2001, Yan et al. 2005).

At 3 dpf, pthlha knockdown led to embryos that had slightly altered sox9a expression in the branchial arches, otic vesicle, and pectoral fin bud (Fig. 6F) and significantly reduced $s o x 9 b$ expression in the tectum, retina, dorsal hindbrain, branchial arches, and pectoral fin bud (Fig. 6G). Knockdown of pthlhb led to $2 \mathrm{dpf}$ embryos with substantially reduced sox $9 a$ expression (most notably in pharyngeal arch 1 and 2, Fig. 6I) and sox $9 b$ expression (retina and branchial arches, Fig. 6J). We conclude that pthlha and pthlhb play a significant role in the regulation of sox $9 a$ and sox $9 b$ at $3 \mathrm{dpf}$ during craniofacial skeleton development.

Sox 9 is a major regulator of chondrogenesis while Runx2 is a major regulator of tooth and bone development (D'Souza et al. 1999, Kim et al. 1999), and in zebrafish, runx $2 b$ is expressed in bone-forming elements (Flores et al. 2006). Morpholino knockdown of pthlha produced animals that overexpressed runx $2 b$ in skeletal elements throughout the craniofacial region (Fig. $6 \mathrm{H}$ ). We conclude that pthlha inhibits or delays runx2b (pthlha $-\|$ runx2b). Similarly, morpholino knockdown of pthlhb produced animals with altered craniofacial morphology and overexpression of $r u n x 2 b$ in skeletal elements and teeth (Fig. 6K). Both endochondral (palatoquadrate, ceratohyal, ceratobranchials-1 to -4 , and especially the tooth-bearing ceratobranchial-5) and dermal bones (opercle and parasphenoid, Mabee et al. (2000)) showed enhanced expression of runx2b. We conclude that pthlha action normally inhibits runx $2 b$ expression in both endochondral and dermal bones and that $\mathrm{MO}$ knockdown of primarily pthlha (and to an extent pthlhb, Fig. 6K) relieves that inhibition.

To understand the role of pthlha and pthlhb during cartilage and bone development, we stained pthlha- and pthlhbknockdown animals with alcian blue for cartilage and alizarin red for mineralized bone. Results showed that 6 dpf pthlha knockdown animals (Fig. 7B) had significantly more alizarin bone staining than controls in the bone collar of the ceratohyal, palatoquadrate, first ceratobranchial, and hyomandibular (Fig. 7A and B, arrows). We conclude that pthlha action inhibits or delays bone mineralization (pthlha $-\|$ bone mineralization). Knockdown of pthlhb (Fig. 7C) caused under- and deformed development of the ceratobranchials and ceratohyals with minimal additional alizarin staining on the palatoquadrate and hyomandibular as controls (Fig. 7A). Double knockdown of pthlha and pthlhb (Fig. 7D) caused substantial deformity along with more alizarin staining than controls. These results are comparable to the murine Pthlh knockout mutants showing premature bone formation (Karaplis et al. 1994). Thus, the finding that pthlha knockdown led to premature bone deposition is consistent with the hypothesis that the function of Pthlh is conserved between zebrafish and mammals and is necessary for chondrogenesis. 

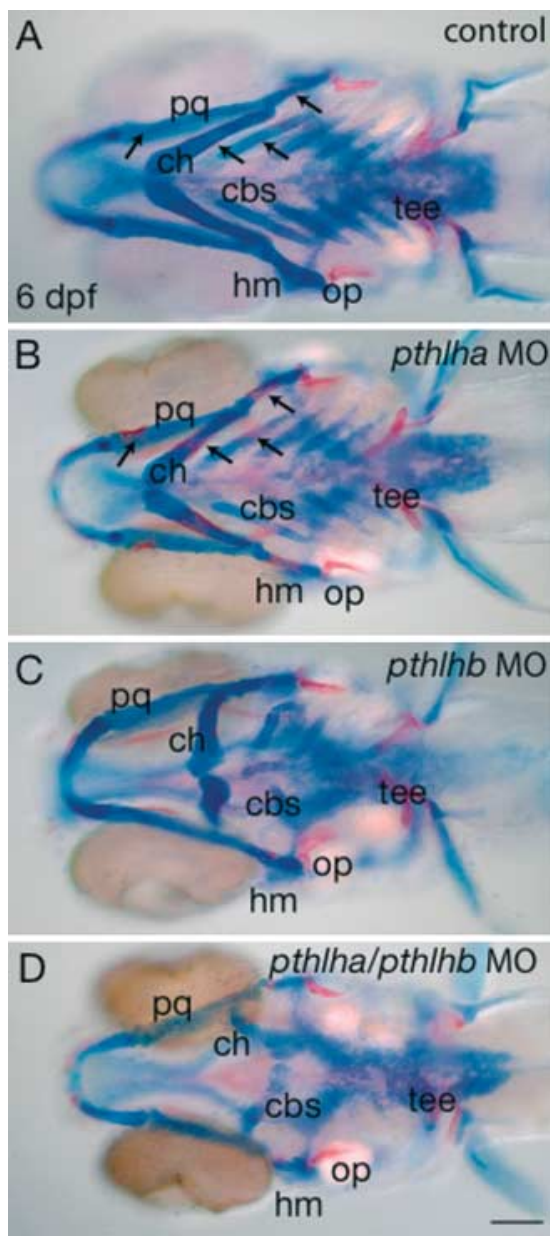

Figure 7 Regulation of pharyngeal arch development by Pth/h co-orthologs at $6 \mathrm{dpf}$. 6 Dpf fish stained with alcian blue and alizarin red. pth/ha MO-treated embryos (B) showed significantly more premature alizarin red staining (bone formation, arrows) compared with noninjected controls (A). pth/hb MO-treated embryos $(C)$ showed deformed pharyngeal skeletons compared with noninjected controls (A) and pthlha knockdown (B). Double knockdown of pth/ha and pth/hb (D) showed severe abnormality in the pharyngeal skeleton. cbs, ceratobranchials; ch, ceratohyal; $\mathrm{hm}$, hyomandibular; op, opercular; pq, palatoquadrate; tee, teeth. Scale bar is $100 \mu \mathrm{m}$.

\section{Discussion}

The zebrafish genome contains two co-orthologs of the human PTHLH gene with pthlha showing more similarity than pthlhb to human PTHLH (Fig. 1A). In mammals, PTHLH is associated with chondrogenesis and development of the pancreas, the craniofacial skeleton, and other tissues (Philbrick et al. 1996). One current hypothesis is that in teleosts, Pthlh is an endocrine hormone that regulates serum calcium homeostasis and osmoregulation. This hypothesis is supported because teleost serum shows high plasma Pthlh levels in heterologous RIAs (Abbink \& Flik 2007) while Pthlh levels are typically low in mammalian plasma. Our hypothesis is that, because Pthlh is expressed during early development, zebrafish Pthlh may have a dual role as a paracrine hormone that is necessary for chondrogenesis and osteogenesis, similar to mammals, and as a circulating hormone for serum calcium homeostasis and osmoregulation.

The partitioning of ancestral subfunctions appears to have occurred in Pthlh co-orthologs (Force et al. 1999, Postlethwait 2007). For example, the NLS of human PTHLH appears to be more strongly conserved in Pthlha than in Pthlhb (Henderson et al. 1995, Nguyen \& Karaplis 1998). Furthermore, Pthlha showed considerable conservation in the $\mathrm{N}$-terminus, midregion, NLS/RNA-binding region, and C-terminus compared with Pthlhb (Miao et al. 2008, Toribio et al. 2010). This situation might be expected under the hypothesis that Pthlha has a conserved functional role in the development of bone and other tissues compared with Pthlhb.

Our phylogenetic analysis showed that proteins encoded by human PTHLH, PTH, and PTH2 form a clade with the relationship ((Pthlh, PTH) PTH2). Analyses of conserved syntenies show that the chromosomal paralogons in which these genes are embedded are as expected if they arose in two rounds of whole genome duplication that occurred at the base of the vertebrate radiation (Dehal \& Boore 2005). The fourth predicted paralog has been lost, which is the most common fate of one member of a pair of duplicated genes, but paralogon analysis suggests that it would likely have been in Hsa1 or Hsa7 (Fig. 1B and C). We conclude that the preduplication ancestor to all vertebrates had a single gene that may have had many of the functions of these three genes today, but that after the vertebrate genome duplications, these genes diversified in function.

Zebrafish have two copies of many human genes that regulate chondrogenesis and craniofacial patterning (for example, RUNX2, SOX9, IHH, BMP2, and MSX2; Ekker et al. 1997, Martinez-Barbera et al. 1997, Yan et al. 2005, Avaron et al. 2006, Flores et al. 2006), and now, as we describe here, PTHLH. These paralogs arose in the TGD (R3) that occurred at the base of the teleost radiation (Amores et al. 1998, Postlethwait et al. 2000, Jaillon et al. 2004, Taylor \& Raes 2004, Nakatani et al. 2007). The TGD produced a pair of zebrafish PTH co-orthologs (Gensure et al. 2004) and a pair of Pthlh co-orthologs that today reside in duplicated chromosome regions.

To evaluate the developmental roles of Pthlha and Pthlhb during chondrogenic and osteogenic developmental programs, we characterized their amino acid composition and conservation, their expression in wild-type and sox9-deficient embryos (Yan et al. 2005, Flores et al. 2006), and their functions by analysis of knockdown animals.

Zebrafish Pthlh co-orthologs were expressed in the developing teeth, pancreas, spinal cord, bone, and cartilage, similar to that of their mammalian orthologs (Clemens et al. 2001), demonstrating a conservation for 450 million years. In the 300 million years since the TGD, pthlha and pthlhb evolved significantly different embryonic expression patterns. The specialization of pthlhb is especially notable because its 
embryonic expression is restricted to chondrocytes at the posterior border of the hyosymplectic and opercular (Fig. 4D and F), while pthlha is expressed in many elements of the pharyngeal skeleton including the ceratobranchials, ceratohyal, and teeth (Figs 3I, M and 4C, G, K). These results show that the pthlh paralog that maintained the most ancestral expression domains (pthlha) is also the one that preserved the most ancestral protein coding domains.

In tetrapods, Ihh secreted by maturing chondrocytes stimulates surrounding perichondrial osteoblasts to secrete Pthlh (Inada et al. 1999). Pthlh diffuses back to prehypertrophic chondrocytes, where, by reciprocal signaling, it binds a receptor (Pth1r or Pth3r), thereby blocking further chondrocyte maturation. Chondroblasts thus continue to proliferate and do not develop to the Ihh-secreting stage, thereby completing a hypothesized negative feedback loop (Vortkamp et al. 1996). Pthlh may slow chondrocyte maturation by inhibiting Runx2 expression via Creb1 (Iwamoto et al. 2003, $\mathrm{Li}$ et al. 2004). Although the process is not yet fully understood, our data show that in pthlha knockdown zebrafish embryos, $r u n x 2 b$ transcript is upregulated, which is consistent with the hypothesis, and that pthlha knockdown leads to premature alizarin staining in the ceratohyal bone collar, suggesting that Pthlha in zebrafish is necessary to slow chondrocyte maturation. Combined, our results suggests that the role of pthlha in zebrafish chondrogenic and osteogenic pathways conserves the role of Pthlh in mammals.

Although previous studies observed Sox 9 expression during retinogenesis (Poché et al. 2008, Yokoi et al. 2009), the robust expression of Pthlh in the retina is a novel finding. Furthermore, Pthlhb expression during retinogenesis was significantly reduced in zebrafish sox $9 b$ mutants, which suggests that retinal expression of Pthlh may be under the control of Sox9 (Fig. 7F, G, I and J). The recent finding that Sox9 is important for development of the retina in teleosts and tetrapods suggests the hypothesis that Sox 9 may exert its effect in eye development at least in part via Pthlh (Poché et al. 2008, Yokoi et al. 2009). Because zebrafish Pthlh co-orthologs showed discrete tissue-specific expression, it is likely that additional novel Pthlh functions may be observed in zebrafish.

The data clearly suggest that pthlh is necessary during the development of neural crest cell-derived craniofacial endochondrogenesis (Figs 6 and 7). Furthermore, zebrafish Pthlh knockdown animals showed a reduction in osx expression in the neurocranium, pharyngeal arches, and pectoral girdle similar to that in humans with campomelic dysplasia (Supplementary Fig. 3, see section on supplementary data given at the end of this article; Yan et al. 2002). The regulation of sox 9 by Pthlh showed considerable conservation between zebrafish and mammals. The increased expression of $r u n \times 2 b$ in knockdown Pthlha animals (Fig. 7) suggests that Pthlh may have a direct role in downregulating run $x 2 b$ expression. Our data are consistent with the hypothesis that Pthlh decreases the expression of $r u n x 2$, which then retards bone mineralization. In the pththa knockdown animals, $r u n \times 2 a$ is upregulated, which leads to premature bone mineralization.
In summary, these studies identified the anciently duplicated teleost orthologs of mammalian Pthlh. Functional studies using mutants and morpholino knockdowns showed that zebrafish pthlh duplicates responded differently to their upstream regulators sox $9 a$ and $s o x 9 b$ and a downstream target run $\times 2 b$ (Supplementary Fig. 4, see section on supplementary data given at the end of this article). These studies lay the groundwork for teasing apart the further functional roles of Pthlh in vertebrate development. Future studies to evaluate the developmental pathways associated with Pthlha and Pthlhb proteins may yield information that would be useful to understand the general rules governing the evolution of duplicated genes.

\section{Supplementary data}

This is linked to the online version of the paper at http://dx.doi.org/10.1530/ JOE-12-0110.

\section{Declaration of interest}

The authors declare that there is no conflict of interest that could be perceived as prejudicing the impartiality of the research reported.

\section{Funding}

National Center for Research Resources (NCRR) grant number 3R01RR020833 ( $\mathrm{J} \mathrm{H} \mathrm{P),} \mathrm{National} \mathrm{Institute} \mathrm{of} \mathrm{Child} \mathrm{Health} \mathrm{and} \mathrm{Human}$ Development grant numbers P01HD22486 (J H P) and HD05034 (D A R), and National Institute of Diabetes and Digestive and Kidney Disease grant number DK60513 (D A R).

\section{Author contribution statement}

All authors contributed to research design, performed research, and contributed to data analyses and to the writing of the manuscript.

\section{Acknowledgements}

This work was covered by Animal Welfare Assurance Numbers A-3009-01 (J H P) and A3762-01 (D A R), IACUC protocols 14-2002 (D A R) and 05-15 RRAA (J H P), 06-09 RRAA (J H P), and Biosafety IBC protocols 11B-2003 and 02B-2008 (D A R). The authors thank grant sponsors: National Center for Research Resources (NCRR) grant numbers R0 RR10715 and R01RR20833, National Institute of Child Health and Human Development grant numbers P01 HD22486 (C K and J H P) and HD05034 (D A R), and National Institute of Diabetes and Digestive and Kidney Disease grant number DK60513 (D A R). They also thank Ruth BreMiller for sectioning. The contents of the paper are solely the responsibility of the authors and do not necessarily represent the official views of grant sponsors.

\section{References}

Abbink W \& Flik G 2007 Minireview: parathyroid hormone-related protein in teleost fish. General and Comparative Endocrinology 152 243-251. (doi:10.1016/j.ygcen.2006.11.010) 
Akiyama H, Kim J-E, Nakashima K, Balmes G, Iwai N, Deng J, Zhang Z, Martin J, Behringer R, Nakamura T et al. 2005 Osteo-chondroprogenitor cells are derived from Sox9 expressing precursors. PNAS 102 14665-14670. (doi:10.1073/pnas.0504750102)

Albertson R, Payne-Fereira T, Postlethwait J \& Yelick P 2005 Zebrafish acvr2a and acvr2b exhibit distinct roles in craniofacial development. Developmental Dynamics 233 1405-1418. (doi:10.1002/dvdy.20480)

Alt B, Reibe S, Feitosa N, Elsalini O, Wendl T \& Rohr K 2006 Analysis of origin and growth of the thyroid gland in zebrafish. Developmental Dynamics 235 1872-1883. (doi:10.1002/dvdy.20831)

Amores A, Force A, Yan Y-L, Joly L, Amemiya C, Fritz A, Ho R, Langeland J, Prince V, Wang Y-K et al. 1998 Zebrafish hox clusters and vertebrate genome evolution. Science 282 1711-1714. (doi:10.1126/science.282. 5394.1711)

Avaron F, Hoffman L, Guay D \& Akimenko M 2006 Characterization of two new zebrafish members of the hedgehog family: atypical expression of a zebrafish Indian hedgehog gene in skeletal elements of both endochondral and dermal origins. Developmental Dynamics 235 478-489. (doi:10.1002/ dvdy.20619)

Bhattacharya P, Yan Y-L, Postlethwait J \& Ruibn D 2011 Evolution of the vertebrate pth2 (tip39) gene family and the regulation of PTH type 2 receptor (pth2r) and its endogenous ligand pth2 by hedgehog signaling in zebrafish development. Journal of Endocrinology 211 187-200. (doi:10.1530/ JOE-10-0439)

Catchen J, Conery J \& Postlethwait J 2009 Automated identification of conserved synteny after whole-genome duplication. Genome Research 19 1497-1505. (doi:10.1101/gr.090480.108)

Chiang EF-L, Pai C-I, Wyatt M, Yan Y-L, Postlethwait J \& Chung B-C 2001 Two Sox9 genes on duplicated zebrafish chromosomes: expression of similar transcription activators in distinct sites. Developmental Biology 231 149-163. (doi:10.1006/dbio.2000.0129)

Clemens T, Cormier S, Eichinger A, Endlich K, Fiaschi-Taesch N, Fischer E, Friedman P, Karaplis A, Massfelder T, Rossert J et al. 2001 Parathyroid hormone-related protein and its receptors: nuclear functions and roles in the renal and cardiovascular systems, the placental trophoblasts and the pancreatic islets. British Journal of Pharmacology 134 1113-1136. (doi:10.1038/sj.bjp.0704378)

Danks J, Ho P, Notini A, Katsis F, Hoffmann P, Kemp B, Martin T \& Zajac J 2003 Identification of a parathyroid hormone in the fish, Fugu rubripes. Journal of Bone and Mineral Research 18 1326-1331. (doi:10.1359/jbmr.2003. 18.7.1326)

Dehal P \& Boore J 2005 Two rounds of whole genome duplication in the ancestral vertebrate. PLoS Biology 3 e314. (doi:10.1371/journal.pbio. 0030314)

Draper B, Morcos P \& Kimmel C 2001 Inhibition of zebrafish fgf8 pre-mRNA splicing with morpholino oligos: a quantifiable method for gene knockdown. Genesis 50 154-156. (doi:10.1002/gene.1053)

D'Souza R, Aberg T, Gaikwad J, Cavender A, Owen M, Karsenty G \& Thesleff I $1999 \mathrm{Cbfa} 1$ is required for epithelial-mesenchyme interactions regulating tooth development in mice. Development 126 2911-2920.

Eisen J \& Smith J 2008 Controlling morpholino experiments: don't stop making sense. Development 135 1735-1743. (doi:10.1242/dev.001115)

Ekker M, Akimenko M, Allende M, Smith R, Drouin G, Langille R, Weinberg E \& Westerfield M 1997 Relationships among msx gene structure and function in zebrafish and other vertebrates. Molecular Biology and Evolution 14 1008-1022. (doi:10.1093/oxfordjournals.molbev. a025707)

Flanagan JA, Power DM, Bendell LA, Guerreiro PM, Fuentes J, Clark MS, Canario AVM, Danks JA, Brown BL \& Ingleton PM 2000 Cloning of the cDNA for the Sea Bream (Sparus aurata) parathyroid hormone-related protein. General and Comparative Endocrinology 118 373-382. (doi:10.1006/ gcen.2000.7481)

Flores M, Lam E, Crosier P \& Crosier K 2006 A hierarchy of runx transcription factors modulate the onset of chondrogenesis in craniofacial endochondral bones in zebrafish. Developmental Dynamics 235 3166-3176. (doi:10.1002/dvdy.20957)
Force A, Lynch M, Pickett FB, Amores A, Yan Y-L \& Postlethwait J 1999 Preservation of duplicate genes by complementary, degenerative mutations. Genetics 151 1531-1545

Garcia-Fernandez J \& Holland P 1994 Archetypal organization of the amphioxus Hox gene cluster. Nature 370 563-566. (doi:10.1038/370563a0)

Gardella T \& Jüppner H 2000 Interaction of PTH and PTHrP with their receptors. Reviews in Endocrine \& Metabolic Disorders 1 317-329. (doi:10.1023/A:1026522619828)

Gensure R, Ponugoti B, Gunes Y, Papasani M, Lanske B, Bastepe M, Rubin D \& Jüppner H 2004 Identification and characterization of two parathyroid hormone-like molecules in zebrafish. Endocrinology 145 1634-1639. (doi:10.1210/en.2003-0964)

Guindon S, Lethiec F, Duroux P \& Gascuel O 2005 PHYML Online - a web server for fast maximum likelihood-based phylogenetic inference. Nucleic Acids Research 33 W557-W559. (doi:10.1093/nar/gki352)

Hanaoka R, Ohmori Y, Uyemura K, Hosoya T, Hotta Y, Shirao T \& Okamoto H 2004 Zebrafish gcmb is required for pharyngeal cartilage formation. Mechanisms of Development 121 1235-1247. (doi:10.1016/j.mod. 2004.05.011)

Henderson J, Amizuka N, Warshawsky H, Biasotto D, Lanske B, Goltzman D \& Karaplis A 1995 Nucleolar localization of parathryoid hormone-related peptide enhances survival of chondrocytes under conditions that promote apoptotic cell death. Molecular and Cellular Biology 15 4064-4075.

Hogan B, Hunter M, Oates A, Crowhurst M, Hall N, Heath J, Prince V \& Lieschke G 2004 Zebrafish $\mathrm{gcm} 2$ is required for gill filament budding from pharyngeal ectoderm. Developmental Biology 276 508-522. (doi:10.1016/j. ydbio.2004.09.018)

Inada $\mathrm{M}$, Yasui T, Nomura S, Miyake S, Deguchi K, Himeno M, Sato M, Yamagiwa H, Kimura T, Yasui $\mathrm{N}$ et al. 1999 Maturational disturbance of chondrocytes in Cbfa1-deficient mice. Developmental Dynamics 214 279-290. (doi:10.1002/(SICI)1097-0177(199904)214:4<279::AIDAJA1 > 3.0.CO;2-W)

Ingleton P, Bendell L, Flanagan J, Teitsma C \& Malment R 2002 Calciumsensing receptor and parathyroid hormone-related protein in the caudal neurosecretory system of the flounder (Platichthys flesus). Journal of Anatomy 200 487-497. (doi:10.1046/j.1469-7580.2002.00036.x)

Iwamoto M, Kitagaki J, Tamamura Y, Gentili C, Koyama E, Enomoto H, Komori T, Pacifici M \& Enomoto-Iwamoto M 2003 Runx2 expression and action in chondrocytes are regulated by retinoid signaling and parathyroid hormone-related peptide (PTHrP). Osteoarthritis and Cartilage 11 6-15. (doi:10.1053/joca.2002.0860)

Jaillon O, Aury J, Brunet F, Petit JL, Stange-Thomann N, Mauceli E, Bouneau L, Fischer C, Ozouf-Costaz C \& Bernot A 2004 Genome duplication in the teleost fish Tetradon nigroviridis reveals the early vertebrate proto-karyotype. Nature 431 946-957. (doi:10.1038/nature03025)

Karaplis AC, Luz A, Glowacki J, Bronson R, Tybulewicz V, Kronenberg HM \& Mulligan RC 1994 Lethal skeletal dysplasia from targeted disruption of the parathyroid hormone-related peptide gene. Genes and Development 8 277-289. (doi:10.1101/gad.8.3.277)

Karperien MC, Lanser P, de Laat SW, Boonstra J \& Defize LHK 1996 Parathyroid hormone related peptide mRNA expression during murine postimplantation development: evidence for involvement in multiple differentiation processes. International Journal of Developmental Biology $\mathbf{4 0}$ 599-608.

Kim I, Otto F, Zabel B \& Mundlos S 1999 Regulation of chondrocyte differentiation by Cbfa1. Mechanisms of Development 80 159-170. (doi:10.1016/S0925-4773(98)00210-X)

Lagerström M, Hellström A, Gloriam D, Larsson T, Schiöth H \& Fredriksson R 2006 The G protein-coupled receptor subset of the chicken genome. PLoS Computational Biology 2 e54. (doi:10.1371/journal.pcbi. 0020054)

Lee K, Deeds JD \& Segre GV 1995 Expression of parathyroid hormone-related peptide and its receptor messenger ribonucleic acid during fetal development of rats. Endocrinology 136 453-463. (doi:10.1210/en.136.2.453)

Li T-F, Dong Y, Ionescu A, Rosier R, Zuscik M, Schwarz E, O'Keefe R \& Drissi H 2004 Parathyroid hormone-related peptide (PTHrP) inhibits Runx2 expression through the PKA signaling pathway. Experimental Cell Research 299 128-136. (doi:10.1016/j.yexcr.2004.05.025) 
Mabee P, Olmstead K \& Cubbage C 2000 An experimental study of intraspecific variation, developmental timing, and heterochrony in fishes. Evolution 54 2091-2106.

Martinez-Barbera J, Toresson H, Rocha SD \& Krauss S 1997 Cloning and expression of three members of the zebrafish Bmp family: Bmp2a, Bmp2b and Bmp4. Gene 198 53-59. (doi:10.1016/S0378-1119(97)00292-8)

Miao D, Su H, He B, Gao J, Xia Q, Zhu M, Gu Z, Goltzman D \& Karaplis A 2008 Severe growth retardation and early lethality in mice lacking the nuclear localization sequence and C-terminus of PTH-related protein. PNAS 105 20309-20314. (doi:10.1073/pnas.0805690105)

Nakatani Y, Takeda H, Kohara Y \& Morishita S 2007 Reconstruction of the vertebrate ancestral genome reveals dynamic genome reorganization in early vertebrates. Genome Research 17 1254-1265. (doi:10.1101/gr.6316407)

Nguyen M \& Karaplis A 1998 The nucleus: a target site for parathyroid hormone-related peptide (PTHrP) action. Journal of Cellular Biochemistry 70 193-199. (doi:10.1002/(SICI)1097-4644(19980801)70:2<193::AIDJCB5 > 3.0.CO;2-J)

Orloff JJ, Ganz MB, Nathanson H, Moyer MS, Kats Y, Mitnick M, Behal A, Gasalla-Herraiz J \& Isales CM 1996 A midregion parathyroid hormonerelated peptide mobilizes cytosolic calcium and stimulates formation of inositol trisphosphate in a squamous carcinoma cell line. Endocrinology 137 5376-5385. (doi:10.1210/en.137.12.5376)

Philbrick WM, Wysolmerski JJ, Galbraith S, Holt E, Orloff JJ, Yang KH, Vasavada RC, Weir EC, Broadus AE \& Stewart AF 1996 Defining the roles of parathyroid hormone-related protein in normal physiology. Physiological Reviews 76 127-173.

Philbrick WM, Dreyer BE, Nakchbandi IA \& Karaplis AC 1998 Parathyroid hormone-related protein is required for tooth eruption. PNAS 95 11846-11851. (doi:10.1073/pnas.95.20.11846)

Pinheiro P, Cardoso J, Gomes A, Fuentes J, Power D \& Canario A 2010 Gene structure, transcripts and calciotropic effects of the PTH family of peptides in Xenopus and chicken. BMC Evolutionary Biology 10 373. (doi:10.1186/ 1471-2148-10-373)

Poché R, Furuta Y, Chaboisser M, Schedl A \& Behringer R 2008 Sox 9 is expressed in mouse multipotent retinal progenitor cells and functions in Müller glial cell development. Journal of Comparative Neurology $\mathbf{5 1 0}$ 237-250. (doi:10.1002/cne.21746)

Postlethwait J 2007 The zebrafish genome in context: ohnologs gone missing. Journal of Experimental Zoology 308B 563-577. (doi:10.1002/jez.b.21137)

Postlethwait J, Woods I, Ngo-Hazelett P, Yan Y-L, Kelly P, Chu F, Huang H, Hill-Force A \& Talbot W 2000 Zebrafish comparative genomics and the origins of vertebrate chromosomes. Genome Research 10 1890-1902. (doi:10.1101/gr.164800)

Provot S \& Schipani E 2005 Molecular mechanisms of endochondral bone development. Biochemical and Biophysical Research Communications $\mathbf{3 2 8}$ 658-665. (doi:10.1016/j.bbrc.2004.11.068)

Robu M, Larson J, Nasevicius A, Beiraghi S, Brenner C, Farber S \& Ekker S 2007 p53 activation by knockdown technologies. PLoS Genetics 3 e78 0787-0801. (doi:10.1371/journal.pgen.0030078)

Rodriguez-Mari A, Yan Y, Bremiller R, Wilson C, Canestro C \& Postlethwait J 2005 Characterization and expression pattern of zebrafish anti-Müllerian hormone (Amh) relative to sox9a, sox $9 b$, and cyp19a1a, during gonad development. Gene Expression Patterns 5 655-667. (doi:10.1016/j.modgep.2005.02.008)

Rohr K \& Concha M 2000 Expression of $n k 2.1 a$ during early development of the thyroid gland in zebrafish. Mechanisms of Development 95 267-270. (doi:10.1016/S0925-4773(00)00345-2)

Rotllant J, Liu D, Yan Y-L, Postlethwait J, Westerfield M \& Du S-J 2008 Sparc (osteonectin) functions in morphogenesis of the pharyngeal skeleton and inner ear. Matrix Biology 27 561-572. (doi:10.1016/j.matbio.2008.03.001)

Sellers R, Luchin A, Richard V, Brena R, Lima D \& Rosol T 2004 Alternative splicing of parathyroid hormone-related protein mRNA: expression and stability. Journal of Molecular Endocrinology 33 227-241. (doi:10.1677/jme.0. 0330227)

Seymour P, Freude K, Dubois C, Shih H, Patel N \& Sander M 2008 A dosagedependent requirement for Sox 9 in pancreatic endocrine cell formation. Developmental Biology 323 19-30. (doi:10.1016/j.ydbio.2008.07.034)
Shor R, Halabe A, Aberbuh E, Matas Z, Fux A, Boaz M \& Wainstein J 2006 PTHrP and insulin levels following oral glucose and calcium administration. European Journal of Internal Medicine 17 408-411. (doi:10.1016/j. ejim.2006.02.019)

Taylor J \& Raes J 2004 Duplication and divergence: the evolution of new genes and old ideas. Annual Review of Genetics 38 615-643. (doi:10.1146/ annurev.genet.38.072902.092831)

Toribio R, Brown H, Novince C, Marlow B, Hernon K, Lanigan L, Hildreth B, Werbeck J, Shu S, Lorch G et al. 2010 The mid-region, nuclear localization sequence, and C terminus of PTHrP regulate skeletal development, hematopoiesis, and survival in mice. FASEB Journal 24 1947-1957. (doi:10.1096/fj.09-147033)

Trivett M, Officer R, Clement J, Walker T, Joss J, Ingleton P, Martin T \& Danks J 1999 Parathyroid hormone-related protein (PTHrP) in cartilaginous and bony fishes. Journal of Experimental Zoology 284 541-548. (doi:10.1002/ (SICI)1097-010X(19991001)284:5 < 541::AID-JEZ10>3.0.CO;2-3)

Valin A, Guillen C \& Esbrit P 2001 C-terminal parathyroid hormone-related protein (PTHrP) (107-139) stimulates intracellular $\mathrm{Ca}^{2+}$ through a receptor different from the type $1 \mathrm{PTH} / \mathrm{PTHrP}$ receptor in osteoblastic osteosarcoma UMR 106 cells. Endocrinology 142 2752-2759. (doi:10.1210/ en.142.7.2752)

Vortkamp A, Lee K, Lanske B, Segre GV, Kronenberg HM \& Tabin CJ 1996 Regulation of rate of cartilage differentiation by Indian hedgehog and PTHrelated protein. Science 273 613-622. (doi:10.1126/science.273.5275.613)

Walker M \& Kimmel C 2007 A two-color acid-free cartilage and bone stain for zebrafish larvae. Biotechnic \& Histochemistry 82 23-28. (doi:10.1080/ 10520290701333558)

Wall D, Fraser H \& Hirsh A 2003 Detecting putative orthologs. Bioinformatics 19 1710-1711. (doi:10.1093/bioinformatics/btg213)

Weir EC, Philbrick WM, Amling M, Neff LA, Baron R \& Broadus AE 1996 Targeted overexpression of parathyroid hormone-related peptide in chondrocytes causes skeletal dysplasia and delayed endochondral bone formation. PNAS 93 10240-10245. (doi:10.1073/pnas.93.19.10240)

Wendelaar Bonga SE \& Pang P 1991 Control of calcium regulating hormones in the vertebrates: parathyroid hormone, calcitonin, prolactin, and stanniocalcin. International Review of Cytology 128 139-213.

Westerfield M 2000 The Zebrafish Book: a Guide for the Laboratory Use of Zebrafish (Danio rerio). Eugene, Oregon: Univeristy of Oregon Press.

Willett C, Zapata A, Hopkins N \& Steiner L 1997 Expression of zebrafish rag genes during early development identifies the thymus. Developmental Biology 182 331-341. (doi:10.1006/dbio.1996.8446)

Wu TL, Vasavada RC, Yang K, Massfelder T, Ganz M, Abbas SK, Care AD \& Stewart AF 1996 Structural and physiological characterization of the mid-region secretory species of parathyroid hormone-related protein. Journal of Biological Chemistry 271 24371-24381. (doi:10.1074/jbc.271. 40.24371)

Wysolmerski JJ, Broadus AE, Zhou J, Fuchs E, Milstone LM \& Philbrick WM 1994 Overexpression of parathyroid hormone-related protein in the skin of transgenic mice interferes with hair follicle development. PNAS 91 1133-1137. (doi:10.1073/pnas.91.3.1133)

Yan Y-L, Miller C, Nissen R, Singer A, Liu D, Kirn A, Draper B, Willoughby J, Morcos P, Amsterdam A et al. 2002 A zebrafish sox 9 gene required for cartilage morphogenesis. Development 129 5065-5079.

Yan Y-L, Willoughby J, Liu D, Crump J, Wilson C, Miller C, Singer A, Kimmel C, Westerfield M, Postlethwait J et al. 2005 A pair of sox: distinct and overlapping functions of zebrafish sox 9 co-orthologs in craniofacial and pectoral fin development. Development 132 1069-1083. (doi:10.1242/dev.01674)

Yokoi H, Yan Y-L, Miller M, BreMiller R, Catchen J, Johnson E \& Postlethwait J 2009 Expression profiling of zebrafish sox 9 mutants reveals that Sox 9 is required for retinal differentiation. Developmental Biology 329 1-15. (doi:10.1016/j.ydbio.2009.01.002)

Received in final form 20 June 2012

Accepted 3 July 2012

Made available online as an Accepted Preprint 3 July 2012 